\title{
Genotyping of nine native Russian cattle breeds combined with the 1000 bull genome project data reveals signatures of selection and adaptation in Russian cattle genomes
}

\author{
A.A. Yurchenko ${ }^{1}$, N.S. Yudin ${ }^{1,2}$, D.M. Larkin ${ }^{1,3 *}$ \\ ${ }^{1}$ Institute of Cytology and Genetics SB RAS, Novosibirsk, Russia \\ ${ }^{2}$ Novosibirsk State University, Novosibirsk, Russia \\ ${ }^{3}$ Royal Veterinary College, University of London, London, UK \\ *e-mail:dmlarkin@gmail.com
}

Key words: cattle native breeds, selection, adaptation, genotyping, sequencing, Russia

Motivation and Aim: In Russian Federation, there are $\sim 20$ native cattle breeds currently being registered. Our previous data suggest that these breeds form four distinct phylogenetic clusters when clustered together with cattle breeds from around the world. In the present study we focused on detecting signatures of adaptation/selection that shaped the Russian cattle genomes during domestication, breed formation, selective breeding, and adaptation to local environments. We combined our genotyping data from nine genetically distinct Russian native cattle breeds with the sequence-based genotypes of four European and two Asian breeds and analysed the combined datasets to spot adaptive genomic changes common among the breeds and those that are characteristics of the Russian breeds only.

Methods and Algorithms: A total of 172 representatives from nine Russian native cattle breeds (ranging 18-30 samples per breed) previously genotyped on the GGP HD150K Bovine SNP array containing $\sim 139 \mathrm{~K}$ SNPs were combined with sequence/HD array genotyping data from six foreign breeds (130 animals total). The data were filtered in Plink and signatures of putative selection in three sets of related breeds were detected separately using the hapFLK software. In parallel, signatures of genomic selection were identified for each breed using the de-correlated composite of multiple signals framework combining the $F_{\mathrm{ST}}, \mathrm{H} 1, \mathrm{H} 12$, Tajima's D and nucleotide diversity statistics. Results: A total of 999 regions under putative selection ranging from $1 \mathrm{bp}$ to $15 \mathrm{Mbp}$ in size were identified in the combined set of all breeds. A total of 1,578 genes were found within these intervals. The strongest putative signals of selection were detected near the genes: LCORL/NCAPG, HMGA2, IMPAD1 (growth), KIT (coat colour), PLAG1 (reproduction). We further detected signatures of selection in Russian breeds related to domestication (KITLG, EDN3, COPA), feed intake (XKR4, TMEM68), milk production (DGAT1, GHR, ABCG2, GLI2, LAP3, TRPV5, FKBP2), and reproduction (CSF2, BCL2, $A N X A 10, N P B W R 1)$. In addition, strong candidate genes for adaptation to cold climate and local environment were found under selection in the Yakut cattle: RETREG1, RPL7, $T N K S$, Kholmogory: AQP5, and multiple Russian breeds: $A R R D C 3, R A D 50, S Y K$.

Conclusion: Multiple regions under putative selection were identified in the set of nine Russian native cattle breeds forming a basis for future genomics-based selection and targeted breeding of Russian cattle.

Acknowledgements: This study was supported by the grant from the Russian Science Foundation (project No. 16-14-00090). 